\title{
Cour du prince et vie religieuse à la fin du Moyen Âge
}

Université de Bourgogne, 14 mai 2003

\section{Vincent Tabbagh}

\section{(2) OpenEdition}

\section{Journals}

Édition électronique

URL : https://journals.openedition.org/cem/904

DOI : $10.4000 /$ cem.904

ISSN : 1954-3093

Éditeur

Centre d'études médiévales Saint-Germain d'Auxerre

Édition imprimée

Date de publication : 15 août 2004

ISSN : 1623-5770

\section{Référence électronique}

Vincent Tabbagh, «Cour du prince et vie religieuse à la fin du Moyen Âge », Bulletin du centre d'études médiévales d'Auxerre / BUCEMA [En ligne], 8 | 2004, mis en ligne le 20 décembre 2006, consulté le 22 septembre 2022. URL : http://journals.openedition.org/cem/904 ; DOI : https://doi.org/10.4000/cem. 904

Ce document a été généré automatiquement le 22 septembre 2022.

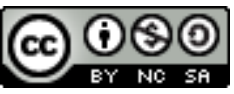

Creative Commons - Attribution - Pas d'Utilisation Commerciale - Partage dans les Mêmes Conditions 4.0 International - CC BY-NC-SA 4.0

https://creativecommons.org/licenses/by-nc-sa/4.0/ 


\section{Cour du prince et vie religieuse à la fin du Moyen Âge}

Université de Bourgogne, 14 mai 2003

\section{Vincent Tabbagh}

1 Il y a quelque paradoxe à vouloir unir ce que tout paraît opposer : la cour n'est-elle pas le lieu même d'une mondanité dont tout le cheminement de la vie religieuse consiste à s'éloigner ? Au XII ${ }^{\mathrm{e}}$ siècle, Jean de Salisbury assimilait celle d'Henri II Plantagenêt à l'Enfer. Comment ce qui est d'abord une manifestation de la puissance seigneuriale et le foyer d'une culture courtoise pourrait-il porter une vie spirituelle spécifique et approfondie ? Cette interrogation animait la demie journée d'études réunissant quelques doctorants et chercheurs de l'Université de Bourgogne le 14 mai 2003, dans le cadre du séminaire de DEA. Partant du constat que dès la fin du XIII ${ }^{e}$ siècle la cour d'un prince est reconnue par la papauté comme institution d'Église susceptible de recevoir des indulgences, et que bien des sources, pour celle de Bourgogne au $\mathrm{XV}^{\mathrm{e}}$ siècle par exemple, montrent l'ampleur des gestes religieux qui s'y déploient, offices, messes, pèlerinages, aumônes, prédications même, les intervenants ont voulu cerner son éventuel rayonnement spirituel, servant de modèle à d'autres milieux en matière de dévotion, mais aussi le rôle des pratiques et des rituels dans son organisation même. La cour en effet, par une sorte d'extension de la chapelle princière ou de l'ordre de chevalerie qu'elle intègre en son sein, peut apparaître comme une communauté ecclésiale ordonnée. La synthèse du palais et du couvent qu'incarne l'Escorial de Philippe II n'est-elle pas déjà présente, comme en germe, dans certaines cours de la fin du Moyen Âge?

2 Logiquement, la cour de Bourgogne a constitué le champ d'observation de quatre sur six des interventions. Sophie Jolivet-Jacquet étudie d'abord les funérailles et cérémonies mémoriales organisées sous Philippe le Bon entre 1430 et 1455, et payées par le duc. Dans les rites du deuil, la cour n'innove pas vraiment : cortèges, luminaires, nombreuses messes. Elle se distingue par une codification peut-être particulièrement rigoureuse, mais variable selon les personnages décédés. Et c'est là que s'introduit une dimension éminemment politique : lorsque meurt en bas âge le fils aîné du duc, Josse, 
les cérémonies sont réduites et prennent un caractère familial et intime nettement marqué. Il n'en va pas de même pour celles où la cour prie pour Isabeau de Bavière en 1435, Jacqueline de Bavière, ou Albert de Habsbourg. Partout, dans ces offices, les représentations symboliques $\mathrm{du}$ défunt tiennent une grande place parmi les manifestations de faste, effigies dont la nature exacte n'est malheureusement pas précisée, ou tableaux d'armoiries par exemple, ceux des chevaliers de la Toison d'Or décédés se drapant de noir lors de la messe des défunts qui accompagne chaque chapitre. La commémoration revêt une signification très particulière à la cour de Bourgogne, mais une signification plus politique ou culturelle que religieuse, exaltant les valeurs propres de l'aristocratie chevaleresque.

D'après les observations de Delphine Lannaud, le sanctuaire de Mont-Roland, près de Dole, où se trouve une statue de la Vierge, tient une place importante dans la piété ducale à l'époque de Philippe le Hardi et Marguerite de Flandre : déplacements de la cour, pèlerinages-vicaires, multiples offrandes, et un peu plus tard quelques fondations. Philippe le Hardi y fait placer ses armes. S'agit-il de la constitution d'un sanctuaire de cour ? En fait le lieu attire depuis longtemps de multiples dévotions et même des sépultures de grandes familles comtoises comme les Chalon, et mène une existence propre en dehors de la cour. On constate cependant dans les années 1440 un dynamisme marqué et un succès certain de ce sanctuaire auprès de foules nombreuses. La cour et les grands semblent ici en avance et peut-être n'est-il pas trop aventureux de parler d'imitation par les simples gens de pratiques qu'ils auraient initiées, bien que le rayonnement nouveau du Mont-Roland dans les années 1440 s'insère sans doute dans un mouvement plus large qui touche d'autres sanctuaires de la région, la collégiale de Vergy par exemple.

Champmol aurait-il alors constitué le vrai sanctuaire de la cour ? Non, démontre Sylvain Faivre : ce monastère ne reçoit que dix donations entre 1389 et 1447 , de la part de roturiers en majorité, donations souvent fragiles, contestées par les héritiers. Les donateurs semblent vouer à la prière pour les morts des biens dont la propriété ne leur était pas pleinement assurée. Seul un lien particulièrement fort avec le duc pousse son chambellan Guy de La Trémoïlle et son maitre d'hôtel Jean Sauvegrain à vouloir y être inhumé, aux pieds de leur maître. Si Champmol reste un mémorial dynastique et ne devient pas le pôle d'une vie religieuse de la cour, il le doit à l'existence antérieure et très solidement établie de la chapelle ducale de Dijon, et au caractère fermé de l'ordre des Chartreux ; ce monastère n'est en rien, pour la cour de Bourgogne, ce que peut être à la même époque celui des Célestins de Paris pour celle de France.

5 La cour comprend de nombreux ecclésiastiques, soigneusement hiérarchisés, régulièrement ou occasionnellement rémunérés, explique Gaëlle Tarbochez. Dès 1165, un chapelain est mentionné, et par la suite quelques chanoines de la chapelle ducale de Dijon assurent le service quotidien du prince avant qu'au $\mathrm{XV}^{\mathrm{e}}$ siècle une chapelle, privée en quelque sorte, se détache de la collégiale dijonnaise devenue une institution pleinement autonome par rapport à la cour. Dans beaucoup de châteaux ducaux réside en permanence au moins un chapelain. L'investissement curial dans le développement du culte s'est fait intense. La chapelle apparaît cependant comme un service parmi d'autres, dont les membres peuvent accomplir des missions diverses sans rapport avec leurs responsabilités liturgiques. L'administration spirituelle de la cour, à laquelle les préséances donnent souvent le premier rang, repose sur une organisation interne complexe, modelée davantage sur les autres services que sur des hiérarchies d'ordres 
sacrés reçus ou le rituel des cérémonies. Mais il faut aussi tenir compte de l'influence du haut personnel ecclésiastique, aumôniers et surtout confesseurs, Laurent Pignon au début du règne de Philippe le Bon par exemple.

6 Dans des démarches qui s'appuient sur les exemples de la cour du roi de Majorque autour de 1300 et de celle de France à la fin du XV siècle, Christophe Cailleaux et Alain Rauwel analysent les enjeux de la présence du saint à la cour. La légitimation qu'il apporte à celle-ci ne peut être maintenue que s'il persévère dans l'ascétisme, pour François de Paule, ou continue son œuvre prédicante et intellectuelle, pour Raymond Lull. Chez ce dernier, comme chez Arnaud de Villeneuve à la même époque, les liens avec la cour n'étouffent-ils pas un prophétisme ou des audaces spirituelles que des personnalités moins socialement reconnues exprimeront plus ouvertement, Marguerite Porète ou Catherine de Sienne ? Raymond Lull a dû affirmer une différence radicale par la revendication de sa propre folie, et François de Paule par la pauvreté et la rigueur effrayantes de son genre de vie. La cour doit également s'ouvrir à l'influence du saint, lui porter aide dans ses entreprises. François de Paule trouve l'appui du roi et d'Anne de Beaujeu pour la fondation de ses couvents de Minimes, et joue un rôle essentiel dans tout le courant de réforme religieuse qui touche le royaume de l'époque. Mais il sert aussi de caution aux entreprises italiennes du roi. Lorsque le prince, puisque sacré, n’a plus à être un saint, il faut un saint à la cour.

7 La vie itinérante des cours les empêche de constituer un pôle spécifique pour leur vie religieuse. Dans le cas bourguignon, le déplacement incessant des chapelains interdit d'organiser un sanctuaire qui remplirait le rôle de la Chapelle de Dijon aux XII ${ }^{e}$ et XIII ${ }^{e}$ siècles. Faute d'un lieu qui lui soit propre, la cour ne parvient pas non plus à rassembler en son sein la commémoration d'un milieu aristocratique aux assises locales et aux choix spirituels dispersés. L'ordre de la Toison d'Or n'assure cette fonction que de manière épisodique. Les ducs Valois n'ont pas privilégié la mémoire spirituelle de la dynastie : ils préférèrent le tombeau à l'obit. La prière du duc et de la cour n'en est pas moins intense, bien que la plupart de ses manifestations restent mal connues, messes et aumônes par exemple. Elle se veut surtout dispensatrice de générosités à de multiples églises ou communautés, sans peut-être même la volonté d'organiser un réseau d'établissements ecclésiastiques soumis à son patronage. La cour de Bourgogne choisit, sous Philippe le Hardi et son petit-fils, de porter une réflexion politique et pastorale, alors que d'autres connaissent davantage la fascination des exemples de sainteté, qu'elle vécut elle-même à l'époque de Colette de Corbie.

\section{INDEX}

Mots-clés : vie religieuse, cour princière 